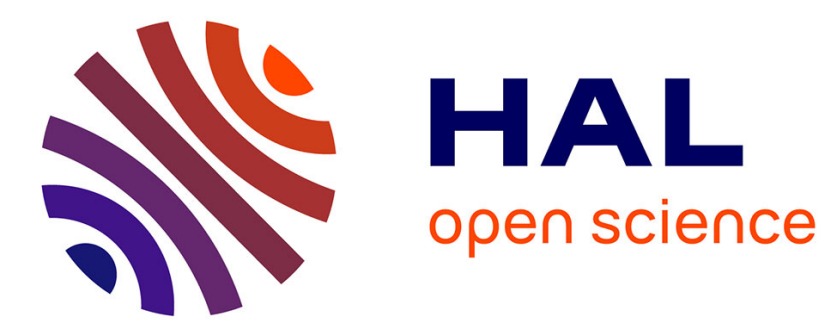

\title{
Les professionnels du droit comme acteurs du politique: revue critique de la littérature nord-américaine et enjeux pour une importation en Europe continentale
} Aude Lejeune

\section{- To cite this version:}

Aude Lejeune. Les professionnels du droit comme acteurs du politique : revue critique de la littérature nord-américaine et enjeux pour une importation en Europe continentale. Sociologie du Travail, 2011, Sociologie du Travail, 53 (2), pp.216-233. 10.4000/sdt.7806 . hal-02399928

\section{HAL Id: hal-02399928 \\ https://hal.univ-lille.fr/hal-02399928}

Submitted on 3 Jan 2022

HAL is a multi-disciplinary open access archive for the deposit and dissemination of scientific research documents, whether they are published or not. The documents may come from teaching and research institutions in France or abroad, or from public or private research centers.
L'archive ouverte pluridisciplinaire HAL, est destinée au dépôt et à la diffusion de documents scientifiques de niveau recherche, publiés ou non, émanant des établissements d'enseignement et de recherche français ou étrangers, des laboratoires publics ou privés.

\section{(1) (1) $\$$}

Distributed under a Creative Commons Attribution - NonCommercial - NoDerivatives| 4.0 
Les professionnels du droit comme acteurs du politique : revue critique de la littérature nord-américaine et enjeux pour une importation en Europe continentale

Lawyers as political actors: Critical review of North-American sociolegal studies and considerations regarding the importation of these works into continental Europe

\section{Aude Lejeune}

\section{CpenEdition}

\section{Journals}

Édition électronique

URL : https://journals.openedition.org/sdt/7806

DOI : $10.4000 /$ sdt.7806

ISSN : $1777-5701$

Éditeur

Association pour le développement de la sociologie du travail

Édition imprimée

Date de publication : 1 juin 2011

Pagination : 216-233

ISSN : 0038-0296

Ce document vous est offert par Université de Lille

\section{Université de Lille}

\section{Référence électronique}

Aude Lejeune, «Les professionnels du droit comme acteurs du politique : revue critique de la littérature nord-américaine et enjeux pour une importation en Europe continentale », Sociologie du travail [En ligne], Vol. 53 - n² 2 | Avril-Juin 2011, mis en ligne le 01 juin 2011, consulté le 03 janvier 2022. URL : http://journals.openedition.org/sdt/7806 ; DOI : https://doi.org/10.4000/sdt.7806

Ce document a été généré automatiquement le 3 janvier 2022.

\section{c)}

Sociologie du travail is licensed under a Creative Commons Attribution-NonCommercial-NoDerivatives 4.0 International License. 


\section{Les professionnels du droit comme acteurs du politique : revue critique de la littérature nord-américaine et enjeux pour une importation en Europe continentale}

Lawyers as political actors: Critical review of North-American sociolegal studies and considerations regarding the importation of these works into continental Europe

Aude Lejeune

Depuis plusieurs décennies, les sciences sociales nord-américaines s'interrogent sur la dimension politique de l'activité des professionnels du droit alors qu'en Europe continentale, droit et politique n'ont été analysés conjointement que très récemment et de façon marginale. Cette différence s'explique par l'histoire spécifique des professionnels du droit aux États-Unis ainsi que par le regard qu'ont porté les sciences sociales sur cet objet ( $1^{\text {re }}$ partie). Si les rapports entre droit et politique constituent un objet de prédilection des Sociolegal studies nord-américaines depuis plusieurs décennies, certains auteurs contemporains affirment que la prise en compte de la dimension politique de l'activité des lawyers ${ }^{1}$ est un phénomène récent. Une revue critique de la production scientifique sur les professionnels du droit en tant qu'acteurs du politique permet de mettre en évidence l'existence de plusieurs courants de recherche qui étudient cette question ainsi que les enjeux scientifiques liés au découpage entre ces différents courants. L'objectif ici n'est pas de présenter ce domaine de recherche foisonnant de manière exhaustive mais plutôt de mettre en exergue une série de travaux qui révèlent les principaux enjeux de réflexion actuels ( $2^{\mathrm{e}}$ partie). La sociologie politique des professionnels du droit ambitionne aujourd'hui de transposer les conclusions éprouvées à l'intérieur des frontières des États-Unis dans d'autres contextes: d'autres époques ou d'autres pays (non démocratiques, en voie de transition 
démocratique, etc.) afin d'étendre la validité de ces conclusions. L'importation de concepts ou de cadres de pensée nord-américains soulève cependant toute une série de questions d'ordres méthodologique et théorique ( $3^{\mathrm{e}}$ partie).

Il est important de souligner que la singularité des lawyers est affirmée par tous les auteurs qui étudient les professions juridiques : l'outil particulier qu'ils mobilisent dans leur activité quotidienne, le droit, empêche de penser ces professions comme tout autre métier mais invite plutôt, à la suite de Max Weber (2007 [1921]), à mettre l'accent sur la spécificité des acteurs qui utilisent le droit parce qu'ils sont porteurs spécialisés de représentations juridiques (Abel et Lewis, 1989). Il existe cependant une grande diversité d'approches au sein des Sociolegal studies. En effet, une partie de cette production scientifique interdisciplinaire émane de juristes désireux de préserver les privilèges liés au statut des professionnels du droit. D'autres travaux, produits par des chercheurs en sciences sociales - sociologues, économistes, politologues ou anthropologues - se révèlent très normatifs parce qu'ils ont été utilisés pour légitimer les transformations proposées par certaines catégories de professionnels du droit. D'autres encore optent pour une posture extrêmement critique à l'égard du fonctionnement de la justice et des professions juridiques. Pour ces raisons, il est fondamental d'être attentif à la position institutionnelle et aux intérêts défendus par les auteurs de ces travaux ainsi qu'aux évolutions de la relation entre le droit et les sciences sociales au cours de l'histoire récente. Par ailleurs, ces différents travaux se réfèrent à plusieurs définitions du droit (law)².

\section{Professionnels du droit, société et sciences sociales aux États-Unis au $X X^{\mathrm{e}}$ siècle}

$3 \mathrm{Si}$, en Europe continentale, les juristes et les sociologues ont élaboré deux sociologies du droit - l'une plus «juridique ", l'autre plus «sociologique » - qui communiquent peu entre elles (Treves, 1966), aux États-Unis, la rencontre entre juristes et chercheurs en sciences sociales est bien plus ancienne (Dezalay et al., 1989). Les Sociolegal studies se sont donc constituées au croisement du droit et des sciences sociales. Il paraît indispensable pour cette raison de les replacer dans leur contexte de production, lié à la constitution de savoirs interdisciplinaires et à l'apparition des sciences sociales au sein des facultés de droit, mais aussi à l'histoire des professionnels du droit et de leur rapport au politique ${ }^{3}$ et, enfin, à l'histoire politique et sociale nord-américaine.

\subsection{La professionnalisation des lawyers et l'autonomie du droit (tournant du $x x^{\mathrm{e}}$ siècle)}

4 Les professions juridiques dans les pays de common law peuvent être réparties en deux catégories : les professionnels du droit indépendants ou praticiens privés d'un côté et les professionnels du droit qui travaillent pour les pouvoirs publics, à savoir les juges,

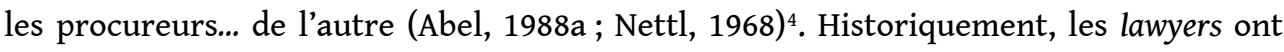
d'abord été des professionnels indépendants avant de devenir des fonctionnaires ou des agents de l'État ${ }^{5}$. Ce n'est qu'à la fin du $\mathrm{xIx}^{\mathrm{e}}$ siècle que cette élite amorce sa professionnalisation, entendue chez Richard L. Abel (1988b) comme un processus de contrôle de l'offre et de la demande de services juridiques en faisant appel à l'intérêt 
général pour justifier ce procédé (Sarfatti Larson, 1977). L'un des traits saillants de ce contrôle de l'offre repose sur l'érection de barrières à l'entrée de la profession qui limitent le nombre d'entrants et opèrent une sélection des candidats. Ce processus conduit à une relative homogénéité des membres de la profession, composée d'hommes blancs, protestants, de milieux sociaux favorisés (les WASP) qui partageaient une culture commune ${ }^{6}$.

Cette époque de construction professionnelle correspond à la création de cursus universitaires autonomes dans les Law schools de l'Ivy league $e^{7}$ de la côté Est reposant sur l'élaboration d'une méthode scientifique d'enseignement du droit prenant appui sur l'analyse de décisions judiciaires, appelée Case method (Harno, 2004 [1953]). Au niveau doctrinal, cette époque marque l'apogée du courant du formalisme juridique qui postule que le droit, en tant que "système complet et cohérent de normes positives " (García Villegas, 2009, p. 33) permet d'expliquer la réalité sociale. En d'autres termes, les penseurs de ce mouvement considèrent que le droit, c'est-à-dire toute réglementation ou législation, peut être appréhendé comme une variable explicative du social. Ce cadre doctrinal appelé Autonomous law se caractérise également par une séparation entre droit et politique et par une disjonction entre la volonté politique et le jugement juridique, puisque ce modèle présuppose une neutralité des décisions judiciaires.

\subsection{New Deal, démocratisation de la profession juridique et Legal realism (années 1930)}

Dans Unequal Justice, Jerold Auerbach (1976) étudie les stratégies apportées par l'élite du barreau en réponse aux changements sociaux qui se produisent au cours $d u x^{e}$ siècle. Il montre précisément que cette élite formée au début $d u x^{e}$ siècle cherche à se structurer en définissant des codes éthiques et des conditions d'entrée dans la profession. Si cette élite parvient entre 1905 et 1925 à restreindre l'accès à la profession aux hommes blancs de milieux sociaux favorisés, elle traverse plusieurs crises dans les décennies qui suivent qui la contraignent à intégrer de nouveaux profils d'avocats aux origines sociales plus diversifiées. La profession connaît ainsi un premier mouvement de démocratisation dans les années 1930 suite à l'arrivée de juristes juifs jusqu'alors tenus à l'écart de l'élite du barreau (Carlin, 1994 [1962]; Carlin et Howard, 1965 ; Dezalay et al., 1989, p. 82).

7 L'arrivée de lawyers juifs au sein des Corporate law firms durant les années du New Deal crée des tensions au sein de la profession. L'indépendance du barreau par rapport au pouvoir politique est alors mise en cause. Dans un contexte de crise économique profonde, accompagnée d'une crise juridique sans précédent, de nombreux débats jurisprudentiels opposent les formalistes à un autre courant doctrinal, le Legal realism (Kalman, 1986 ; Schlegel, 1995) ${ }^{8}$. Ce dernier s'impose finalement et exerce une influence considérable sur la culture juridique nord-américaine à partir de 1930. Les réalistes proposent d'appréhender les rapports entre droit et société d'une manière tout à fait inédite. Avec eux, le droit cesse d'être un savoir scientifique autonome pour devenir le produit d'actions humaines, guidées par la poursuite d'intérêts ou de valeurs. Suivant cette idée, le réalisme s'attache à mettre en avant le caractère politique des décisions judiciaires (Miller, 2009) ${ }^{9}$. Par ailleurs, le modèle traditionnel de l'autonomie du droit prôné par les formalistes est ainsi mis en cause au profit d'une vision instrumentale du 
droit qui renvoie à une intégration des aspirations juridiques et politiques afin que le droit réponde aux besoins sociaux (modèle de la Sociological jurisprudence, appelé plus tard Responsive law) (Nonet et Selznick, 2001, p. 51 ; Shamir, 1995, p. 135-149). Le droit est alors envisagé comme un outil de transformation du social et du politique.

Ces débats doctrinaux s'accompagnent d'un accroissement de la légitimité du droit en tant qu'outil d'aide à la décision politique (Vauchez, 2001) et d'une exigence nouvelle de prise en compte de savoirs issus des sciences sociales, notamment en ce qui concerne l'impact des actions juridiques sur le contexte social dans lequel elles s'inscrivent (Wiebe, 1967).

\subsection{Droit, changement social et naissance du Law \& Society Movement (années 1960-1970)}

9 Dans les années 1960 aux États-Unis, dans le cadre du mouvement des droits civiques, des Civil Rights Acts et de la War on poverty lancée par le Président Johnson ${ }^{10}$, et suite à l'émergence du Legal realism dans les années 1930, le droit est considéré comme un exceptionnel moteur de réduction des inégalités qui doit permettre de changer la société. Dans ce contexte, les lawyers sont perçus comme les acteurs du changement d'une société nouvelle à construire, tel que l'exprime l'introduction du rapport de la conférence nationale Law \& Poverty qui se tient à Washington DC en 1965 : «Dans une société de lois, ce sont les professionnels du droit qui sont à l'origine de toute action sociale. Historiquement, la profession juridique a d'ailleurs été à l'avant plan dans tous les grands mouvements nationaux de réformes publiques [...] Dans ces batailles à venir, le barreau doit assumer légitimement son rôle de leader ${ }^{11}$.

10 C'est dans cette mouvance politique et intellectuelle marquée par le réalisme juridique que naît en 1964 le mouvement Law \& Society. Juristes et sociologues s'y rassemblent autour d'un projet commun de transformation profonde de l'éducation juridique par l'intégration dans les cursus de droit d'outils et de cadres de réflexions de sciences sociales (Abel, 1995 ; Erlanger, 2005 ; Friedman, 1986 ; Hunt, 1993 ; Lipson et Wheeler, 1986 ; Trubek, 1990 ; Vauchez, 2001). Né dans les Law schools du Mid-West et de la côté Ouest - et non dans les écoles reconnues de la côte Est -, ce mouvement ambitionne de révolutionner les savoirs juridiques dans un contexte où le droit est envisagé comme un outil qui doit être mis au service d'une meilleure justice sociale (Galanter, 1974 ; Garth, 1980; Rosenthal, 1974). Pour cela, l'une des préoccupations majeures des sciences sociales est d'analyser les écarts potentiels entre le droit, le Law in books, et son application concrète dans la société, le Law in action, dans le cadre de ce qui a été appelé les Gaps studies, paradigme dominant de l'époque ${ }^{12}$. Les sciences sociales ambitionnent également de mettre en évidence l'influence de l'économie, de la politique ou du social sur le Law in books (Scheingold, 2004 [1974]; Macaulay et al., 2007). L'intégration de chercheurs de sciences sociales dans le champ de la recherche Law \& Society s'accompagne de l'établissement d'une division du travail entre, d'une part, les juristes qui, détenant une position privilégiée, restent les seuls à être considérés comme légitimes pour énoncer le "discours scientifique sur le social et le commentaire légitime sur le droit » (Dezalay et al., 1989, p. 84) et, d'autre part, les nouveaux arrivants qui tentent de construire leur place dans ce champ de recherche.

11 À la même époque, au sein de la profession, la légitimité des élites et la foi en l'autorité juridique sont très fortement ébranlées. Les lawyers sont alors contraints d'accepter 
d'intégrer dans leurs rangs de nouveaux profils, notamment des femmes ou des afro-américains (Auerbach, 1976). Cette deuxième vague de démocratisation de l'accès à la formation et aux professions juridiques contribue à l'augmentation du nombre d'étudiants en droit et à la création de nouvelles Law schools ${ }^{13}$. Cependant, l'ouverture de la profession ne conduit pas à une égalité entre ses membres. De nombreuses études de l'époque soulignent les clivages entre les juristes en fonction du lieu de formation, des conditions d'exercice du métier et du type de pratique. Les avocats solo practitioners dans des contextes urbains connaissent des conditions difficiles d'exercice (Carlin, 1994 [1962]) alors que les firmes multinationales offrent de meilleures conditions de travail (Smigel, 1964). Une corrélation est ainsi établie entre les facultés dans lesquelles sont formés les avocats et l'environnement de travail. En effet, les domaines du droit considérés comme les plus prestigieux, les affaires, sont toujours réservés à une élite provenant des écoles les plus reconnues ${ }^{14}$ (Heinz et Laumann, 1982 ; Nelson et Sandefur, 2005).

\subsection{Les Critical legal studies et la mise en cause de la dichotomie Droit/Société (années 1980-1990)}

12 La crise culturelle et économique de la seconde moitié des années 1970 aux États-Unis contribue à mettre en cause le fonctionnement de l'État-providence. À la même époque, les cours fédérales américaines deviennent plus conservatrices et la concurrence sur le marché juridique s'accroît (Scheingold, 2004 [1974]). Ces différents facteurs contribuent à affaiblir la croyance dans le potentiel du droit pour changer la société (Carle, 2005), ainsi que le résume la présidente de la Law \& Society Association dans les années 1990 : « Désormais, ce n'est plus évident que le droit puisse contribuer à produire une société plus juste. Je pense que la plupart d'entre nous [.. .] s'interrogent sur le potentiel du droit dans ce monde de plus en plus fragmenté, déchiré ${ }^{15}$. Dans ce contexte, les Critical legal studies suggèrent d'opérer un dépassement par rapport aux recherches menées dans le cadre du Law \& Society Movement (LSM). Selon ces critiques, l'alternative proposée par le Legal realism dans les années 1930 n'a pas remis en cause la scission fondamentale entre droit et société qui est au cœur de la pensée formaliste. Même si leurs travaux soulignent les écarts entre les prescriptions juridiques et la réalité que ces règles tentent de façonner, les réalistes partent du principe que le droit peut contribuer de manière significative à l'émergence d'une justice sociale (Kessler, 1995). Le réalisme juridique a donc entretenu une conception du droit et de la société comme deux entités distinctes et autonomes comme l'indiquent Susan S. Silbey et Austin Sarat : «Parce que nous [chercheurs du LSM] avons été occupés à problématiser la relation entre le droit et la société, [...] nous avons été attentifs aux liens qui se tissaient entre droit et société en partant de l'idée que ces deux entités étaient séparées et singulières $»^{16}$. Dans les années 1980 , ces travaux critiques proposent quant à eux d'envisager le droit comme constitutif de la société, c'est-à-dire en soulignant son ancrage dans la vie sociale, et non pas comme une entité distincte qui dominerait la société et tenterait de la réguler depuis l'extérieur (Abel, 1995; Garth et Sarat, 1998 ; Hunt, 1993 ; Sarat et Simon, 2003 ; Silbey, 1985 ; Silbey et Sarat, 1987). Ils contribuent ainsi à une relativisation de l'unité et de la cohérence du droit.

Le courant récent des Legal consiousness studies s'inscrit dans cette veine de mise en cause de la dualité droit/société en cherchant à « déceler la présence et les effets du 
droit dans les relations sociales [et] les différentes façons dont les individus ordinaires expérimentent et comprennent le juridique lorsqu'ils invoquent le droit et les significations juridiques, les évitent ou leur résistent " (Ewick et Silbey, 1997, p. 34). Susan S. Silbey et Patricia Ewick, dont l'ouvrage The Common Place of Law de 1998 (Ewick et Silbey, 1998) constitue l'une des références majeures de ce courant (Israël et Pélisse, 2004), indiquent: "le comportement n'est donc pas simplement "modelé" par le juridique puisqu'il existe en dehors du droit tout en étant influencé par lui de même que l'on ne peut pas dire que les comportements échappent à l'emprise du juridique. "Droit" et "Société" existent l'un dans l'autre et sont en relation l'un avec l'autre " (Brigham, 2000 ; García Villegas, 2003 ; Greenhouse et al., 1994 ; Merry, 1990 ; Ewick et Silbey, 1997, p. 101).

14 La légitimation progressive des sciences sociales au sein des facultés de droit a contribué à faire émerger un savoir interdisciplinaire orienté vers la compréhension de l'effectivité et les effets du droit sur le monde social. À partir des années 1980, les auteurs critiques adoptent une posture réflexive à l'égard du mouvement Law \& Society et élaborent une épistémologie critique de leur propre discipline (Vauchez, 2001). C'est dans ce contexte que sont nés plusieurs courants de sociologie des professions juridiques.

\section{La sociologie politique des lawyers : courants récents et controverses}

15 Alors que les juges ont été analysés depuis plusieurs décennies par les réalistes comme des acteurs politiques, certains auteurs affirment que les lawyers ont été tenus à l'écart de ce type d'analyse et suggèrent aujourd'hui d'ouvrir « la boîte noire des lawyers » afin de s'interroger sur leur impact sur le monde social (Mather, 2009). Ils tentent par-là de dépasser une sociologie des professions juridiques focalisée sur les caractéristiques internes des professions, telles que les carrières, les parcours ou les inégalités au sein de la communauté (Friedman, 1989). Cette relative nouveauté de la prise en compte du rôle politique des lawyers doit être interrogée parce qu'elle représente un enjeu scientifique relatif aux découpages des frontières entre les courants de recherches concurrents. Trois courants paraissent incontournables pour un bilan de l'état actuel de la réflexion : le Political lawyering, le Cause lawyering et le Judicial politics ${ }^{17}$.

Un premier ensemble de travaux, regroupés principalement au sein d'ouvrages collectifs dirigés par Terence Halliday, co-directeur du centre de recherche Law \& Globalization de l'American Bar Foundation de Chicago, et Lucien Karpik, professeur à l'École nationale supérieure des Mines de Paris (Halliday et Karpik, 1997 ; Halliday et al., 2007), souhaite contribuer à la récente redécouverte du politique (Commaille, 2009; Scheingold, 1999). L'étude du Political lawyering s'interroge sur l'impact de la profession d'avocat dans la promotion du libéralisme politique. Elle montre qu'au cours de l'histoire, une fraction importante d'avocats s'est à plusieurs reprises - et parfois de manière virulente - opposée à l'État. En ce sens, les avocats ont mené des actions collectives de type politique visant une transformation du droit et de l'État et ont été très attachés à défendre les droits et libertés fondamentaux des individus face à l'État. Ces auteurs envisagent donc la profession d'avocat comme "un sujet doté d'une capacité d'action stratégique qui, loin de se cantonner au pur exercice de la fonction judiciaire, loin de manifester passivement les effets des grandes déterminations 
sociales, a tracé délibérément son chemin » (Karpik, 1995, p. 12). Selon ces auteurs, le Political lawyer défend des droits de l'homme de première génération liés à la liberté et à l'exercice de la citoyenneté politique (Vasak, 1978) et bénéficie d'une grande légitimité au sein de la profession. En effet, «ces avocats politiques se rattachent à une longue histoire, à une philosophie politique riche et diverse, à une expérience politique diversifiée, à une justification qui n'a guère besoin d'être perpétuellement renouvelée tant elle va de soi » (Karpik, 2008, p. 284).

17 Certains auteurs critiquent le caractère idéaliste de cette conception du rôle politique des avocats, comme l'illustre le commentaire d'Yves Dezalay à propos de l'ouvrage Les avocats de L. Karpik (1995) : « la thèse est séduisante. Peut-être même trop. Ce triomphe de la vertu, même s'il est entrecoupé de quelques périodes de déclin, fait trop songer à une histoire enchantée. Du moins telle que pourrait la rêver l'Ordre des avocats " (Bancaud et al., 1997, p. 69; Abel, 1998). Ces critiques portent également sur le fait que les auteurs du Political lawyering ne s'intéressent qu'aux revendications des représentants des barreaux ou de groupes d'avocats qui prétendent parler au nom de la profession dans son ensemble. En effet, le Political lawyering met l'accent sur ce qui fait consensus au sein de la profession d'avocats - le Civic professionalism ${ }^{18}$ - et ne prend donc pas en considération les revendications plus minoritaires ou marginales des professionnels du droit.

18 Le Cause lawyering, deuxième courant d'analyse nord-américain, s'intéresse quant à lui à l'action d'une minorité d'avocats qui utilisent l'arène judiciaire pour défendre des causes politiques (Sarat et Scheingold, 1998, 2001, 2005). Ces professionnels du droit, définis comme « des avocats qui usent de leurs talents et des ressources qui sont à leur disposition pour atteindre des objectifs politiques et sociaux plutôt que d'assumer la fonction traditionnelle de représentation des intérêts de leurs clients " (Sarat et Scheingold, 2003, p. 31), se situent dans une position de rejet de la conception classique du professionnalisme juridique fondée sur le postulat de neutralité et revendiquent d'exercer leur métier au nom de causes politiques. Ce courant de recherche analyse ainsi deux dimensions à première vue difficilement conciliables de l'action des professionnels du droit : celle du traitement juridique d'un cas et celle de la défense militante d'une cause, celle de l'expert et de l'engagé politique ${ }^{19}$. La plupart de ces théories de l'engagement politique des professionnels du droit considèrent que les activités militantes concernent une partie des avocats, les autres membres de la profession étant «non engagés ». Or, S. Scheingold et A. Bloom nuancent cette distinction et suggèrent d'envisager l'engagement sur le mode du continuum allant des formes les moins transgressives aux activités les plus en rupture avec la norme professionnelle et la pratique dominante (Krishnan, 2003 ; Scheingold et Bloom, 1998) ${ }^{20}$.

19 Le Judicial politics, troisième courant présenté ici dont les travaux sont beaucoup plus hétérogènes que ceux des deux précédents courants, est né dans les écoles de sciences politiques et analyse le rôle politique des cours et tribunaux (Epp, 1998 ; Miller, 2009). Depuis peu, certains auteurs s'interrogent sur l'intervention des lawyers dans le processus judiciaire. Lynn Mather (2009) suggère d'envisager le travail quotidien des juristes en tant qu'activité politique en dépassant la distinction entre avocats engagés et avocats conventionnels qu'elle ne juge pas heuristique. Elle considère plutôt que le fait que certains professionnels n'affichent pas ouvertement leur engagement ou leur orientation politique ne veut pas dire qu'ils n'essaient pas de défendre certains idéaux et valeurs à travers leur activité professionnelle. Les juristes sont ainsi envisagés 
comme des acteurs du politique dans la mesure où le processus de traduction en termes juridiques d'un problème exprimé par un citoyen profane en termes ordinaires contribue à redéfinir ce problème. Bien plus que de simples intermédiaires à travers lesquels passe un problème juridique, les lawyers ajoutent au problème de leur client leurs propres idées, objectifs et valeurs (Harrington, 1994). Ainsi, l'intervention du professionnel du droit ne peut se résumer à un ensemble de techniques mais inclut au contraire toute une série de choix qui sont contingents et incertains (Kilwein, 1998) ${ }^{21}$. Cette dernière perspective d'analyse invite à appréhender le professionnel du droit comme une boîte noire que le chercheur a pour mission d'ouvrir et de déchiffrer afin d'expliquer le chemin entre les inputs (les demandes de clients) et les outputs (les décisions judiciaires).

Chacun de ces courants récents tente de marquer sa spécificité en définissant de manière singulière le rapport qu'entretiennent les juristes au politique. Les auteurs du Political lawyering critiquent l'approche du politique des Cause lawyers, considérant que ces derniers négligent l'étude des libertés fondamentales et se concentrent sur des droits spécifiques, ceux des minorités. Les deuxièmes reprochent aux premiers d'adopter une perspective strictement institutionnelle du politique et des professions juridiques, négligeant ainsi les inégalités dans les rapports de pouvoir ou dans les relations avec les élites. Les derniers mettent en cause l'amalgame des partisans du Cause lawyering entre rôle politique et engagement militant. Ces controverses témoignent des enjeux scientifiques, politiques et institutionnels liés à la définition des rapports entre droit et politique.

21 Au-delà de ces débats d'écoles, ce regain d'intérêt pour le politique dans l'étude des professions juridiques et, dans ce cas, des lawyers, est lié au contexte nord-américain dans lequel ces travaux sont produits. Ils offrent de nouvelles opportunités pour contribuer aux débats actuels sur les rapports entre société civile et État, la montée de valeurs néolibérales ou la globalisation des processus juridiques. Cette réflexion invite à s'interroger sur les enjeux scientifiques et politiques liés à l'internationalisation des conclusions nord-américaines dans un contexte de globalisation.

\section{Phénomène local ou global ? Enjeux scientifiques de l'internationalisation des Sociolegal Studies nord-américaines}

Les études nord-américaines des cours et tribunaux ont depuis longtemps mis l'accent sur la dimension politique alors que ce n'est pas le cas en Europe où le droit et le politique ont été considérés comme deux champs séparés de recherche. Comme l'indique Mark Miller, professeur de science politique nord-américain : «Pour certains, penser ensemble droit et politique se révèle difficile, spécialement pour ceux qui sont familiers avec l'approche du droit et de la justice des pays d'Europe continentale [...]. Cependant, les chercheurs en science politique qui étudient les cours et tribunaux aux États-Unis admettent facilement que notre système a toujours mélangé et entrecroisé ces deux concepts $»^{22}$.

L'un des enjeux actuels majeurs de la sociologie nord-américaine des professions juridiques consiste à étendre les conclusions éprouvées à l'intérieur des frontières nationales dans d'autres contextes politiques. Parmi ces tentatives 
d'internationalisation, on peut évoquer, entre autres, l'ouvrage Fighting for Political Freedom (Halliday et al., 2007) qui élargit la portée du Political lawyering aux pays non occidentaux ou l'utilisation par de nombreux auteurs des analyses faites de la Cour suprême américaine pour comprendre d'autres cours dans d'autres pays. L'on mentionnera également la transposition par A. Sarat et S. Scheingold de leur cadre d'analyse dans des contextes très variés de régimes démocratiques et non démocratiques (Sarat et Scheingold, 2005) ou encore l'article de Martin Shapiro et Alec Stone Sweet sur les nouvelles politiques constitutionnelles en Europe, qui commence ainsi: «Longtemps considérée comme une particularité nord-américaine, la Constitutional judicial review est aujourd'hui un phénomène global» (Alter, 1994; Maveety, 2009 ; Shapiro et Stone Sweet, 1994, p. 397). Dans un contexte de globalisation, l'internationalisation des résultats nord-américains représente bel et bien un enjeu scientifique inédit pour les sciences sociales aux États-Unis.

Soucieux d'offrir un panorama international, les travaux américains sont généralement fondés sur des études comparatives portant sur plusieurs pays. La plupart de ces travaux ont cependant tendance à présupposer la généralisation des conclusions nord-américaines et à négliger les spécificités nationales au profit d'une certaine uniformité. Ils ambitionnent par-là de démontrer la validité internationale de la théorie énoncée au départ (Commaille et Dumoulin, 2009 ; Kawar, 2011 ; Rueschemeyer, 1989). Les analyses de cas révèlent ainsi des différences qui invitent à rester attentif aux particularités des systèmes juridiques, des cadres nationaux et des contextes locaux pour analyser le rôle politique des professionnels du droit (Ferrari, 1990; Schwartz, 2006; Trevin o, 1996) ainsi que leur relation à l'État (Abel, 1985; Abbott, 1986, 1988; Boigeol et Willemez, 2005 ; Fielding et Portwood, 1980 ; García Villegas, 2010). C'est à cette prudence qu'invitent S. Silbey et A. Sarat : "Nous [chercheurs du LSM] devons être attentifs à ne pas considérer nos observations sur le droit comme universelles. Au contraire, nous devons porter une attention particulière aux spécificités des situations ${ }^{23}$. Quels sont, en effet, les facteurs qui doivent être pris en compte pour comprendre les spécificités nationales dans le cas de l'importation des conclusions nord-américaines en Europe continentale ${ }^{24}$ ?

Contrairement à l'idée largement répandue selon laquelle la prise en compte de la culture juridique des différents pays suffit à expliquer les relations entre droit et société qui existent au sein de chacun d'entre eux (Merryman, 1969), plusieurs travaux contemporains suggèrent de mettre en exergue des facteurs d'ordre politique (García Villegas, 2009). Trois dimensions paraissent ainsi fondamentales et interdépendantes: la culture juridique de civil ou de common law, les rapports entre les professionnels du droit et les pouvoirs publics et, enfin, les différents types d'État. Ces facteurs permettent à la fois d'analyser les spécificités des pays d'Europe continentale par rapport aux États-Unis et de faire ressortir les divergences entre États européens, afin d'affiner l'analyse comparative.

26 La culture juridique constitue un premier facteur à prendre en considération dans le cadre d'une analyse du rôle politique des lawyers. Les courants de recherche intéressés par ces questions sont nés dans des pays de common law davantage ouverts aux changements établis par la jurisprudence que dans les pays de code, où la primauté est accordée aux sources formelles du droit. Dans le premier cas, le juge énonce le droit, dans le second, il est «bouche de loi » (Parson, 1954). Aux États-Unis, le processus à travers lequel les magistrats de la Cour suprême ont la possibilité de se prononcer sur 
la constitutionnalité des lois selon un processus de Constitutional review (Shapiro et Stone Sweet, 1994) confère à ceux-ci un grand pouvoir par rapport aux pouvoirs exécutif et législatif. Ainsi, «dans les pays de common law, [...] la relative autonomie des cours permet aux cause lawyers, comme aux professionnels du droit en général, d'utiliser le processus juridique en tant qu'outil politique [...]. Dans les pays de droit civil, les cours sont moins indépendantes et plus réticentes à protéger et à étendre les droits, ou à empiéter sur les prérogatives qui appartiennent à l'État. Il en résulte que les frontières entre droit et politique sont plus clairement marquées que dans les pays de droit commun $»^{25}$. Bien que la distinction entre systèmes de droit commun et de droit civil soit critiquée par certains, elle est considérée par la plupart des comparatistes comme heuristique (Serverin, 2000). Plusieurs points distinguent ces deux systèmes juridiques. La règle de droit est mobilisée dans les pays de common law pour répondre à un cas précis et a vocation à acquérir ensuite une validité d'ordre général, alors que, dans les pays de droit continental, elle est élaborée par le législateur pour régir l'ensemble des cas qui pourraient se présenter. Ensuite, le raisonnement juridique et la position du juge diffèrent par le fait que, d'un côté, le magistrat construit la règle de droit, dans les limites des contraintes posées par la jurisprudence, en fonction du cas spécifique qui lui est confronté alors que de l'autre, il se réfère aux catégories générales préétablies (Serverin, 2000). Enfin, bien que la promotion et la garantie des droits et libertés individuels aient été défendues par les Révolutions française et américaine, chacune d'elles a élaboré une conception singulière des rapports entre les individus et l'État: dans les pays de common law, les professionnels du droit ont pour mission de protéger les individus face à l'arbitraire de l'autorité absolue alors que dans les pays de civil law, la protection de ces droits et libertés individuels est garantie par l'État. Ces distinctions suggèrent que le raisonnement juridique des professionnels du droit dans les pays de common law leur permet une plus grande marge d'autonomie dans la transformation du droit. Actuellement, peu d'études à visée comparative portent sur les voies, judiciaire ou législative, à travers lesquelles les professionnels du droit sont susceptibles de mobiliser le droit. Ce domaine de recherches encore largement inexploré permettrait pourtant d'interroger le phénomène d'accroissement du pouvoir judiciaire par rapport aux pouvoirs exécutif et législatif (Commaille et Dumoulin, 2009) attestés par de nombreux auteurs contemporains (Hirschl, 2004; Tate et Vallinder, 1995).

27 Le deuxième élément qui permet de souligner les spécificités nationales, lié au premier, repose sur les rapports qu'entretiennent les professionnels du droit avec l'État. Dans son analyse comparative des champs juridiques et des courants critiques de sciences sociales en France et aux États-Unis, Mauricio García Villegas (2009) montre que la question de la souveraineté, qui s'est posée en termes contrastés dans les pays de droit commun et dans ceux de droit civil, est centrale pour comprendre les rapports qui s'établissent entre les professionnels du droit et le pouvoir politique. En France, où le droit exprime la souveraineté de l'État, les professionnels du droit sont les dépositaires du savoir de l'État, les «serviteurs de l'État » (Nettl, 1968) ou les «méta-garants de la raison juridique " (Commaille, 2000). Historiquement, les premiers professionnels du droit apparaissent d'ailleurs au sein de l'appareil d'État et non pas, comme dans les pays de common law, sur le marché (Abel, 1988a). Dans les pays de code, la profession d'avocat s'est développée sur le modèle de la corporation, dans lequel les barrières à l'entrée du métier permettent à ses membres de préserver la pureté de l'activité juridique et de s'autoproclamer gardiens du temple du droit (Bourdieu, 1986 ; Dezalay, 
1990). Dans cette conception du droit comme émanation de l'État, le Code représente l'incarnation de la souveraineté populaire et de la volonté générale. Toute contestation $\mathrm{du}$ droit est donc difficile parce qu'elle implique la contestation de cette volonté générale. En revanche, aux États-Unis et dans les autres pays de common law, le droit précède toute organisation politique et la loi appartient au peuple. Le pouvoir politique est donc contraint de respecter les droits individuels. Ainsi, « le droit positif - à la différence du droit naturel ou des droits fondamentaux - est bien une manifestation de ce pouvoir politique et, comme tel, peut être l'objet d'une critique juridique institutionnalisée, qui s'est développée à l'intérieur du système juridique sans remettre en question le contrat social»(García Villegas, 2009, p. 49). Suite à l'influence du réalisme juridique, les professionnels du droit sont plutôt pensés comme des 《ingénieurs sociaux $»^{26}$ qui utilisent le droit pour répondre aux problèmes qui se posent dans la société nord-américaine; leur légitimité repose sur leur capacité à mobiliser les sciences sociales et à travailler sur un marché.

Ces rapports entre professionnels du droit et État peuvent être mis en relation avec les différents types d'État, ce qui constitue un troisième facteur de différenciation entre les États-Unis et les pays d'Europe continentale (Badie et Birnbaum, 1982). Ces «types d'État " reposent sur la distinction proposée par Alexis de Tocqueville (1981 [1835]) dans De la Démocratie en Amérique entre État faible et État fort. Les États-Unis représenteraient un modèle du premier type d'État dans lequel «la société y agit par elle-même et sur elle-même. Il n'existe de puissance que dans son sein »; la France illustre de manière exemplaire le second modèle d'État, dans lequel « l'État atteint tout d'un coup les extrêmes limites de sa force, tandis que les particuliers se laissent tomber en un moment jusqu'au dernier degré de faiblesse » (Birnbaum, 1993, p. 163). Dans la mesure où le modèle nord-américain d'organisation juridique et étatique a largement influencé l'orientation des recherches de sciences sociales, D. Rueschemeyer montre qu'il est logique que les travaux produits dans ce contexte ne se soient intéressés que de manière secondaire à la dimension étatique. S'appuyant sur les travaux d'Ira Katznelton et Kenneth Prewitt, il avance que l'État américain est moins tangible, plus diffus et plus influencé par des acteurs non-étatiques que les États européens (Katznelton et Prewitt, 1979). De plus, la constitution américaine n'établit pas d'État qui définirait le bien commun mais met plutôt en place une économie politique dans laquelle le bien commun est le résultat de l'agrégation de préférences privées (Rueschemeyer, 1989) ${ }^{27}$. La dimension étatique et la constitution spécifique du pouvoir politique constituent donc des variables explicatives des pratiques et de la capacité d'action collective des professionnels du droit.

Depuis plusieurs décennies, les sciences sociales ambitionnent de comparer les professionnels du droit en fonction des systèmes juridiques dans lesquels ils exercent leur métier (Barceló et Cramton, 1999 ; Clark, 1999, 2002). La sociologie des professions juridiques s'est ainsi tour à tour focalisée sur les caractéristiques communes aux différentes occupations dans le cadre d'une perspective fonctionnaliste, puis, avec un regard interactionniste, aux tâches exercées au quotidien par ces professionnels. Elle a ensuite comparé les intérêts des professionnels, montrant par-là que leurs stratégies et actions visaient à garantir la pérennisation d'intérêts économiques et corporatistes, dans le cadre de travaux dits du market control, inspirées des courants marxistes et néowébériens (Saks, 1983). La plupart de ces études omettent dans bien des cas de mettre en relief l'influence de variables sociopolitiques sur les rapports tant entre droit et politique qu'entre professionnels du droit et pouvoir politique (Abel, 1985). Plus 
récemment, certains auteurs - majoritairement aux États-Unis mais aussi dans le reste du monde - ont proposé de se focaliser sur les objectifs que poursuivent les professionnels du droit, en analysant le comportement des professionnels et en observant ce qu'ils font ou tentent de faire collectivement (Burrage, 1988; Friedman, 1989 ; Heger Boyle, 2000 ; Whittington et al., 2008.). En s'intéressant à l'engagement collectif des professionnels du droit au nom d'idéaux politiques, cette perspective de recherches ouvre la voie à de nouvelles analyses attentives à la dimension étatique, et plus largement politique, des processus juridiques et judiciaires.

\section{Conclusion}

Les sciences sociales nord-américaines s'intéressent depuis longtemps à la dimension politique de l'activité juridique. Déjà au début du $\mathrm{xx}^{\mathrm{e}}$ siècle lors de la constitution des lawyers en tant que profession, il était commun d'affirmer que les professionnels du droit jouaient un rôle politique et que leurs actions et décisions étaient influencées par des valeurs ou des intérêts. Sur le vieux continent, ce type de propos paraissait encore difficilement concevable jusqu'il y a peu, ce qui s'explique par plusieurs facteurs mis en exergue ici.

Les évolutions de la profession de lawyer et de ses rapports à la société et à la doctrine juridique dans la société nord-américaine ont permis d'appréhender les conditions spécifiques de production des Sociolegal studies au cours du $\mathrm{xx}^{\mathrm{e}}$ siècle. Alors que le formalisme juridique considérait le droit comme autonome par rapport au politique, il a été envisagé par la doctrine réaliste nord-américaine comme un outil de transformation de l'ordre social et politique. Aujourd'hui, bien que le rôle du droit dans l'émergence d'une plus grande justice sociale soit mis en cause par plusieurs courants critiques, le réalisme a profondément marqué la conception nord-américaine des relations entre droit et politique. Les travaux récents de sociologie des professions juridiques s'intéressent aux mobilisations collectives et à l'engagement des lawyers au nom d'idéaux politiques : certains traitent du rôle des professionnels dans l'émergence et l'expansion du libéralisme politique, d'autres se penchent sur le rôle des avocats dans la défense de causes politiques, d'autres encore analysent la pratique du droit comme un processus de traduction. Tous s'inscrivent dans une réflexion globale sur les rapports entre la société civile et les pouvoirs publics dans un contexte de globalisation $\mathrm{du}$ droit et des pratiques juridiques où l'internationalisation des conclusions nordaméricaines au-delà des frontières nationales représente un enjeu scientifique de première envergure ${ }^{28}$. Ces cadres de pensées et catégories d'analyses sont le produit de leur contexte contingent et particulier dont sont retracées ici les principales caractéristiques dans une perspective de sociologie politique historique. Leur transposition dans d'autres pays nécessite donc de prendre en considération les spécificités nationales et locales, à savoir la culture juridique, les rapports que les professionnels du droit entretiennent avec les pouvoirs publics et les différents types d'État. Ces réflexions permettent d'ouvrir la voie à de nouvelles pistes de recherche à l'intersection des questions soulevées par la sociologie politique, la sociologie du droit, la sociologie des professions et la sociologie des mobilisations collectives. 


\section{BIBLIOGRAPHIE}

Abbott, A., 1986. Jurisdictional Conflicts: A New Approach to the Development of the Legal Professions. ABF Research Journal 11 (2), 187-224.

Abbott, A., 1988. The System of Professions: An Essay on the Division of Expert Labor. University of Chicago Press, Chicago.

Abel, R.L., 1985. Law Without Politics: Legal Aid Under Advanced Capitalism. UCLA Law Review 32, 474-642. Abel, R.L., 1988a. Lawyers in the Civil Law World. In: Abel, R., Lewis, P. (Eds.), Lawyers in Society: The Civil Law World. University of California Press, Berkeley/Los Angeles/ Oxford, pp. 1-53.

Abel, R.L., 1988b. United States: The Contradiction of Professionalism. In: Abel, R., Lewis, P. (Eds.), Lawyers in Society: The Common Law World. University of California Press, Los Angeles/Oxford, pp. 186-243.

Abel, R.L., 1995. Law and Society Reader. New York University Press, New York.

Abel, R.L., 1998a. Lawyers for Liberalism: Axiom, Oxymoron, or Accident? Books on Law, Book Reviews, pp. 9-15.

Abel, R.L., 1998b. Speaking Law to Power: Occasions for Cause Lawyering. In: Sarat, A., Scheingold, S. (Eds.), Cause Lawyering: Political Commitments and Professional Responsibilities. Oxford University Press, New York, pp. 69-117.

Abel, R.L., Lewis, P., 1988a. Lawyers in Society: The Common Law World. University of California Press, Berkeley/Los Angeles/Oxford.

Abel, R.L., Lewis, P., 1988b. Lawyers in Society: The Civil Law World. University of California Press, Berkeley/Los Angeles/Oxford.

Abel, R.L., Lewis, P., 1989a. Putting Law Back into the Sociology of Lawyers. In: Abel, R., Lewis, P. (Eds.), Lawyers in Society: Comparative Theories. University of California Press, Los Angeles, pp. $478-526$.

Abel, R.L., Lewis, P., 1989b. Lawyers in Society: Comparative Theories. University of California Press, Los Angeles.

Abel, R.L., Lewis, P., 1996. Lawyers in Society: An Overview. University of California Press, Berkeley/Los Angeles/Oxford.

Alter, K., 1994. Judicial Politics in the European Community: European Integration and the Pathbreaking Cassis of Dijon Decision. Comparative Political Studies 26 (4), 535-561.

Auerbach, J., 1976. Unequal Justice. Lawyers and Social Change in Modern America. Oxford University Press, New York.

Badie, B., Birnbaum, P., 1982 [1979]. Sociologie de l'État. Grasset, Paris.

Bancaud, A., Dezalay, Y., Salais, R., Karpik, L., 1997. Symposium sur Les avocats. Entre l'État, le public et le marché, $\mathrm{XIII}^{\mathrm{e}}-\mathrm{XX}^{\mathrm{e}}$ siècle. Sociologie du Travail 39 (1), 63-90.

Barceló, J., Cramton, R., 1999. Lawyers Practice and Ideals: A Comparative View. Kluwer, New York. 
Birnbaum, P., 1993. Mouvements sociaux et types d'États : vers une approche comparative. In : Chazel, F. (Ed.), Action collective et mouvements sociaux. Puf, Paris, pp. 163-176.

Boigeol, A., Willemez, L., 2005. Fighting for Survival: Unification, Differentiation and Representation of the French Bar. In: Felstiner, W. L. (Ed.), Reorganisation and Resistance. Legal Profession Confront a Changing World, Oxford/Portland Oregon, 41-65.

Bourdieu, P., 1986. La force du droit. Éléments pour une sociologie du champ juridique. Actes de la recherche en sciences sociales 64, 3-19.

Brigham, J., 2000. The Constitution of Interests: Beyond the Politics of Rights. New York University Press, New York/Londres.

Burrage, M., 1988. Revolution and the Collective Action of the French, American, and English Legal Professions. Law \& Social Inquiry 13 (2), 225-277.

Cain, M., 1979. The General Practice Lawyer and the Client: Towards a Radical Conception. International Journal of the Sociology of Law 7, 331-354.

Carle, S., 2005. Lawyers' Ethics and the Pursuit of Social Justice: A Critical Reader. New York University Press, New York/Londres.

Carlin, J., 1994 [1962]. Lawyers on Their Own: The Solo Practitioner in an Urban Setting. Austin and Winfield Publishers, San Francisco.

Carlin, J., Howard, J., 1965. Legal Representation and Class Justice. UCLA Law Review 12, 381-437.

Clark, D., 1999. Comparing the Work and Organization of Lawyers Worldwide: The Persistence of Legal Traditions. In: Barceló, J., Cramton, R. (Eds.), Lawyers Practice and Ideals: A Comparative View. Kluwer, New York, pp. 9-155.

Clark, D., 2002. Legal Education and the Legal Profession. In: Clark, D., Ansay, T. (Eds.), Introduction of the Law of the United States. Kluwer, Deventer/Boston, pp. 13-34.

Commaille, J., 2000. Territoires de justice : une sociologie politique de la carte judiciaire. Puf, Paris.

Commaille, J., 2009. Recension de l'ouvrage Fighting for Political Freedom: Comparative Studies of the Legal Complex and Political Liberalism de T., Halliday, L., Karpik, M. Feeley, 2007. L'Année sociologique 59 (2), 449-456.

Commaille, J., Dumoulin, L., 2009. Heurs et malheurs de la légalité dans les sociétés contemporaines. La sociologie politique de la « judiciarisation ». L'Année Sociologique 59 (1), 63 107.

Conley, J., Baker, S., 2005. Review Essay: Fall from Grace or Business as Usual? A Retrospective Look at Lawyers on Wall Street and Main Street. Law and Social Inquiry 30, 83-821.

Sousa Santos de, B., Rodríguez Garavito, C., 2005. Law and Globalization from Below: Towards a Cosmopolitan Legality. Cambridge University Press, Cambridge.

Dezalay, Y., 1990. Juristes purs et marchands de droit. Division du travail de domination symbolique et aggiornamento dans le champ du droit. Politix 3 (10), 70-91.

Dezalay, Y., Sarat, A., Silbey, S., 1989. D’une démarche contestataire à un savoir méritocratique. Esquisse d'une histoire sociale de la sociologie juridique américaine. Actes de la recherche en sciences sociales $78,79-93$. 
Ellmann, S., 1998. Cause Lawyering in the Third World. In: Sarat, A., Scheingold, S. (Eds.), Cause Lawyering: Political Commitments and Professional Responsibilities. Oxford University Press, New York, pp. 349-430.

Epp, C., 1998. The Rights Revolution: Lawyers, Activists. and Supreme Courts in Comparative Perspective. University of Chicago Press, Chicago/Londres.

Erlanger, H., 2005. Organizations, Institutions, and the Story of Shmuel: Reflections on the 40th Anniversary of the Law and Society Association. Law and Society Review 39 (1), 1-10.

Ewick, P., Silbey, S., 1997. Devant la loi : la construction sociale du juridique. In : KourilskyAugeven, C. (Ed.), Socialisation juridique et conscience du droit : attitudes individuelles, modèles culturels et changement social. LGDJ, Paris, pp. 33-56.

Ewick, P., Silbey, S., 1998. The Common Place of Law: Stories from Everyday Life. University of Chicago Press, Chicago. Felstiner, W., 2005. Reorganisation and Resistance: Legal Profession Confront a Changing World. Hart Publishing, Oxford/Portland Oregon.

Ferrari, V., 1990. Developing Sociology of Law. A World-Wide Documentary Enquiry. Giuffre, Milan.

Fielding, A., Portwood, D., 1980. Professions and the State: Towards a Typology of Bureaucratic Professions. Sociological Review 28, 23-53.

Friedman, L., 1986. The Law and Society Movement. Stanford Law Review 38, 763-780.

Friedman, L., 1989. Lawyers in Cross-Cultural Perspective. In: Abel, R.L., Lewis, P. (Eds.), Lawyers in Society: Comparative Theories. University of California Press, Berkeley/Los Angeles/Oxford, pp. 1-26.

Galanter, M., 1974. Why the "Haves" Come out Ahead: Speculations on the Limits of Legal Change. Law and Society Review 9, 95-160.

García Villegas, M., 2003. Symbolic Power without Symbolic Violence? Critical Comment of Legal Consciousness Studies in USA. Droit et Société 53, 137-163.

García Villegas, M., 2009. Champs juridiques et sciences sociales en France et aux États-Unis. L'Année sociologique 59 (1), 29-62.

García Villegas, M., 2010. Sociología y crítica del derecho. Fontamará, México.

Garth, B., 1980. Neighborhood Law Firms for the Poor: A Comparative Study of the Recent Developments in Legal Aid and in Legal Profession. Sijthoff/Noordhoff, Alphen aan den Rijn/ Maryland.

Garth, B., Sarat, A., 1998. Justice and Power in Socio-legal Studies: Fundamental Issues in Law and Society. Northwestern University Press, Evanston.

Greenhouse, C., Yngvesson, B., Engel, D., 1994. Law and Community in Three American Towns. Cornell University Press, Ithaca, NY.

Halliday, T., 1987. Beyond Monopoly: Lawyers, State Crisis, and the Professional Empowerment. University of Chicago Press, Chicago/Londres.

Halliday, T., Karpik, L., 1997. Lawyers and the Rise of Political Liberalism: Europe and North America from the Eighteenth to Twentieth Centuries. Oxford University Press, New York.

Halliday, T., Karpik, L., Feeley, M., 2007. Fighting for Political Freedom: Comparative Studies of the Legal Complex and Political Liberalism. Hart Publishing, Oxford/Portland Oregon. 
Harno, A., 2004 [1953]. Legal Education in the United States: A Report Prepared for the Survey of the Legal Profession. Lawbook Exchange, Clark.

Harrington, C., 1994. Outlining a Theory of Legal Practice. In: Cain, M., Harrington, C. (Eds.), Lawyers in a Postmodern World: Translation and Transgression. New York University Press, New York, pp. 206-226.

Heger Boyle, E., 2000. Is Law the Rule? Using Political Frames to Explain Cross-National Variation in Legal Activity. Social Forces 79 (2), 385-418.

Heinz, J., Laumann, E., 1982. Chicago Lawyers: The Social Structure of the Bar. Russell Sage Foundation/American Bar Foundation, New York/Chicago.

Hirschl, R., 2004. Towards Juristocracy: the Origins and Consequences of the New Constitutionalism. Harvard University Press, Cambridge.

Hunt, A., 1993. Explorations in Law and Society: Toward a Constitutive Theory of Law. Routledge, New York/London.

Huyse, L., 1996. Legal Experts in Belgium. In: Abel, R.L., Lewis, P. (Eds.), Lawyers in Society: An Overview. University of California Press, Berkeley/Los Angeles/Oxford, pp. 169-187.

Israël, L., 2001. Usages militants du droit dans l'arène judiciaire : le cause lawyering. Droit et Société 49, 793-824. Israël, L., 2009. L'arme du droit. Presses de Sciences Po, Paris.

Israël, L., Pélisse, J., 2004. Quelques éléments sur les conditions d'une « importation ». Note préliminaire à la traduction du texte de S. Silbey et P. Ewick. Terrains et travaux 6, 101-111. Kalman, L., 1986. Legal Realism at Yale. University of North Carolina Press, Chapel Hill. Karpik, L., 1995. Les avocats, Entre l'État, le public et le marché, XIII ${ }^{\mathrm{e}}-\mathrm{XX}^{\mathrm{e}}$ siècle. Gallimard, Paris. Karpik, L., 2008. Les professions libérales sont-elles solubles dans le marché ? In : Le Bianic, T., Vion, A. (Eds.), Action publique et légitimités professionnelles. LGDJ, Paris, pp. 279-288.

Katznelton, I., Prewitt, K., 1979. Constitutionalism, Class, and the Limits of Choice in US Foreign Policy. In: Fagen, R. (Ed.), Capitalism and the State in US-Latin American Relations. Stanford University Press, Palo Alto, CA, pp. 25-40.

Kawar, L., 2011. Legal Mobilization on the Terrain of the State: Creating a Field of Immigrant Rights Lawyering in France and the United States. Law and Social Inquiry, à paraître.

Kessler, M., 1995. Lawyers and the Social Change in the Postmodern World. Law and Society Review 29 (4), 769-789.

Kilwein, J., 1998. Still Trying: Cause Lawyering for the Poor and the Disadvantaged in Pittsburgh, Pennsylvania. In: Sarat, A., Scheingold, S. (Eds.), Cause Lawyering: Political Commitments and Professional Responsibilities. Oxford University Press, New York, pp. 181-200.

Krishnan, J., 2003. Cause Lawyering en transgression dans un pays en développement : le cas de l'Inde. Droit et Société 55, 629-658.

Lipson, L., Wheeler, S., 1986. Law and the Social Sciences. Russell Sage Foundation, New York.

Macaulay, S., Friedmann, L., Mertz, E., 2007. Law in Action. A Sociolegal Reader. Foundation Press, s.l.

Mather, L., 2009. Bringing the Lawyers Back In. In: Miller, M. (Ed.), Exploring Judicial Politics. Oxford University Press, New York, pp. 48-63. 
Maveety, N., 2009. Comparative Judicial Studies. In: Miller, M. (Ed.), Exploring Judicial Politics. Oxford University Press, New York, pp. 294-306.

Merry, S., 1990. Getting Justice and Getting Even: Legal Consciousness among Working-Class Americans. University of Chicago Press, Chicago.

Merry, S., 1995. Resistance and the Cultural Power of Law. Law and Society Review 29, 11-26. Merryman, J., 1969. The Civil Law Tradition: An Introduction to the Legal Systems of Western Europe and Latin America. Stanford University Press, Palo Alto, CA.

Miller, M., 2009. Exploring Judicial Politics. Oxford University Press, New York.

Nelson, R., Sandefur, R., 2005. United States, From Professional Dominance to Organizational Dominance: Professionalism, Inequality, and Social Change Among Chicago Lawyers, 1975-1995. In: Felstiner, W. (Ed.), Reorganisation and Resistance, Legal Profession Confront a Changing World. Hart Publishing, Oxford/Portland Oregon, pp. 313-342.

Nelson, R., Trubek, D., 1992. Introduction. In: Nelson, R., Trubek, D., Solomon, R. (Eds.), Lawyers' Ideals/Lawyers' Practices. Cornell University Press, Ithaca, NY, pp. 1-28.

Nettl, J., 1968. The State as a Conceptual Variable. World Politics 20 (4), 559-592.

Nonet, P., Selznick, P., 2001. Toward Responsive Law. Law and Society in Transition. Transactions Publishers, New Brunswick.

Parson, T., 1954. Essays in Sociological Theory. "A Sociologist Looks at the Legal Profession". Free Press, New York.

Pound, R., 1967. Interpretations of Legal History. Peter Smith, Gloucester.

Rosenthal, D., 1974. Lawyer and client: Who is in charge? Russell Sage Foundation, New York.

Roussel, V., 2002. Affaires de juges. Les magistrats dans les scandales politiques en France. La Découverte, Paris.

Rueschemeyer, D., 1973. Lawyers and Their Society: A Comparative Study of the Legal Profession in Germany and in the United States. Harvard University Press, Cambridge.

Rueschemeyer, D., 1989. Comparing Legal Professions: A State-Centered Approach. In: Abel, R.L., Lewis, P. (Eds.), Lawyers in Society: Comparative Theories. University of California Press, Los Angeles, pp. 289-321.

Saks, M., 1983. Removing the Blinkers? A critique of Recent Contributions to the Sociology of Professions. Sociological Review 31, 1-21.

Sarat, A., Scheingold, S., 1998. Cause Lawyering: Political Commitments and Professional Responsibilities. Oxford University Press, New York.

Sarat, A., Scheingold, S., 2001. Cause Lawyering and the State in Global Era. Oxford University Press, New York.

Sarat, A., Scheingold, S., 2003. Quelques éclaircissements sur l'invention du Cause lawyering. Entretien réalisé et traduit par L. Israël. Politix 16 (62), 31-37.

Sarat, A., Scheingold, S., 2005. The Worlds Cause Lawyers Make: Structure and Agency in Legal Practice. Stanford University Press, Palo Alto, CA.

Sarat, A., Simon, J., 2003. Cultural Analysis, Cultural Studies, and the Law: Moving Beyond Legal Realism. Duke University Press, Durham. 
Sarfatti Larson, M., 1977. The Rise of Professionalism: a Sociological Analysis. University of California Press, Berkeley/Los Angeles/Londres.

Scheingold, S., 1999. Taking Weber Seriously: Lawyers, Politics, and the Liberal State. Law and Social Inquiry 24 (4), 1061-1081.

Scheingold, S., 2004 [1974]. The Politics of Rights: Lawyers. Public Policy and Political Change. Yale University Press, New Haven/Londres.

Scheingold, S., Bloom, A., 1998. Transgressive Cause Lawyering: Practice Sites and the Politicization of the Professional. International Journal of the Legal Professions 5, 209-254.

Scheingold, S., Sarat, A., 2004. Something to Believe In: Politics, Professionalism, and Cause Lawyering. Stanford University Press, Palo Alto, CA.

Schlegel, J.H., 1995. American Legal Realism and Empirical Social Science. University of North Carolina Press, Chapel Hill.

Schwartz, R., 2006. Law, Society and Democracy: Comparative Perspectives. Sage, New York. Serverin, E., 2000. Sociologie du droit. La Découverte, Paris.

Shamir, R., 1995. Managing Legal Uncertainly: Elite Lawyers in the New Deal. Duke University Press, Durham.

Shapiro, M., Stone Sweet, A., 1994. The New Constitutional Politics of Europe. Comparative Political Studies 26 (4), 397-420.

Silbey, S., 1985. Ideals and Practices in the Study of Law. Legal Studies Forum 9, 7-22.

Silbey, S., Sarat, A., 1987. Critical Traditions in Law and Society Research. Law and Society Review $21(1), 165-174$.

Smigel, E., 1964. The Wall Street Lawyer: Professional Organization Man? Free Press of Glencoe, New York.

Tate, C.N., Vallinder, T., 1995. The Global Expansion of Judicial Power. New York University Press, New York.

Tocqueville (de), A., 1981 [1835]. De la démocratie en Amérique. Garnier-Flammarion, Paris.

Treves, R., 1966. La Sociologia del diritto. Edizioni di Comunità, Milan.

Trevin o, J., 1996. The Sociology of Law: Classical and Contemporary Perspectives. Saint Martin's Press, New York.

Trubek, D., 1990. Back to the Future: The Short Happy Life of the Law and Society Movement. Florida State University Law Review 18 (1), 4-68.

Vasak, K., 1978. Les dimensions internationales des droits de l'homme. Unesco, Paris.

Vauchez, A., 2001. Entre droit et sciences sociales. Retour sur l'histoire du mouvement Law and Society. Genèses 45, 134-149.

Wald, P., 1965. Law and Poverty. Report to the National Conference on Law and Poverty, Washington DC.

Weber, M., 2007. [1921]. Sociologie du droit. Puf, Paris.

Whittington, K., Kelemen, D., Caldeira, G., 2008. The Oxford Handbooks of Political Science. Oxford University Press, Oxford.

Wiebe, R., 1967. The Search for Order (1977-1920). Hill and Wang Editions, New York. 
Willemez, L., 2003. Engagement professionnel et fidélité militante. Les avocats travaillistes dans la défense judiciaire des salariés. Politix 16 (62), 145-164.

\section{NOTES}

1. Nous utilisons le terme anglo-saxon lawyer parce qu'il n'existe aucune traduction de ce terme en français. Professionnel du droit qui peut conseiller ses clients et les défendre devant les juridictions, il peut être praticien privé ou employé par un organisme public, dans le cadre de l'aide légale par exemple. De plus, le terme lawyer sous-entend une certaine unité, un sentiment d'appartenance à un groupe professionnel commun qui n'existe pas chez les professionnels du droit des pays de droit civil où les métiers juridiques sont divisés, segmentés (Huyse, 1996).

2. Le terme law aux États-Unis et au Royaume-Uni, bien que traduit par «droit", n'a pas exactement la même signification. Si, de l'autre côté de l'Atlantique, le terme law tend à ne faire référence qu'au droit positif, le terme « droit » comprend à la fois le droit positif et l'idée de juste (García Villegas, 2009).

3. L'étude des pratiques et caractéristiques des professionnels du droit permet d'affiner la compréhension des systèmes juridiques (Rueschemeyer, 1973 ; Abel, 1988b ; Abel et Lewis, 1988a, 1988b, 1989a, 1989b, 1996).

4. Dietrich Rueschemeyer distingue quant à lui quatre sous-groupes parmi les professionnels du droit, différemment représentés du côté américain et du côté européen : judiciary, lawyers in legal practice, lawyers in government service, lawyers in private employment (Rueschemeyern 1973, p. 30).

5. En revanche, dans les pays de civil law, l'élite des professionnels du droit se constitue d'abord au sein de l'État avant que n'apparaissent des professionnels du droit indépendants.

6. Cette relative homogénéité due à la seule présence de WASP ne doit pas faire oublier les disparités qui existent entre les avocats issus des écoles réputées et exerçant dans des Corporate law firms et ceux issus d'écoles moins prestigieuses.

7. L'Ivy league est composée de huit universités privées de la côte Est qui comptent parmi les plus anciennes du pays : Brown, Columbia, Cornell, Harvard, Yale, etc.

8. Alors que le réalisme juridique a exercé une grande influence sur la doctrine nord-américaine, du côté européen, les thèses anti-formalistes ne sont pas parvenues à s'imposer de manière décisive (García Villegas, 2009).

9. L'une des branches du Legal realism appelée Behavioralism s'est d'ailleurs attachée à montrer que les décisions judiciaires peuvent être analysées et prédites en fonction des intérêts et valeurs des juges.

10. Quelques mois après l'assassinat du président Kennedy, la War on poverty est lancée par le président Johnson en 1964 pour lutter contre les difficultés économiques et un taux très élevé de pauvreté. Concrètement, le Congrès des États-Unis approuve l'Economic opportunity act qui conduit à la création de l'office of economic opportunity dont la mission est de procéder à une gestion locale au niveau des états des budgets fédéraux consacrés à la lutte contre la pauvreté.

11. "In a society of laws, it is the lawyers who catalyze the social action. Historically, the legal profession has been in the forefront of every great national movement for public reform. [...] In the critical battles to come, the Bar must assume its rightful role of leader » (Wald, 1965, p. 1).

12. Inspirés par le Legal realism traduit en termes sociologiques, ces Gap studies considèrent le droit comme une variable dépendante, par opposition à la vision formaliste du droit.

13. Ce contexte favorise la reconnaissance des actions menées par les avocats qui s'attachent à la promotion d'une justice sociale (Scheingold et Sarat, 2004).

14. Même si les études plus récentes semblent amoindrir ce constat, elles continuent à souligner les écarts entre les différents praticiens du droit (Conley et Baker, 2005). 
15. «It is no longer clear that law can produce a more just society. I think many of us [...] wonder about the possibilities for law in this increasingly fractioned world (Merry, 1995, p. 12-13).

16. «Because we have been busy problematizing the relationship between law and society, [...] we looked for the connections between law and society as if the two were separate and singular. They are not. "The law" is a fiction, but laws are real. Similarly, "society" is a fiction » (Silbey et Sarat, 1987, p. 172).

17. L'ordre de présentation de ces courants ne doit pas laisser supposer une évolution chronologique dans la mesure où la production scientifique de chaque «école » influence et est en même temps influencée par les réflexions des autres courants de recherche.

18. Dans Beyond Monopoly, T. Halliday expose : «l'existence d'intérêts sectoriels parmi les lawyers permet d'expliquer les luttes au sein de la profession. Ces intérêts ne jouent cependant pas un rôle significatif dans la définition des valeurs intrinsèques de la profession [...]. Au-delà de tous conflits d'intérêt particuliers, il existe des intérêts qui dépassent même la profession, c'est le professionnalisme civique » (Halliday, 1987, p. 369-370).

19. L'une des particularités du Cause lawyering est d'analyser cet engagement tel qu'il s'exprime dans la pratique quotidienne des professionnels alors que la plupart des travaux mettent en avant le militantisme qui se manifeste en dehors de l'activité professionnelle (Roussel, 2002). L'ouvrage fondateur de ce courant de recherche, The Politics of Rights, écrit par S. Scheingold en 1974, offrait une première étude de l'action des avocats en tant que moteurs du changement politique et social (Scheingold, 2004 [1974]).

20. Ces catégories accordent beaucoup d'importance à l'environnement et aux conditions d'exercice de la profession, ce facteur étant jugé déterminant dans la manière dont les avocats conçoivent leur rôle et leur rapport au politique (Nelson et Trubek, 1992). L'influence de ce courant en France a ainsi permis de poser la question des conditions d'importation et d'internationalisation de ces travaux (Israël, 2001, 2009; Willemez, 2003).

21. Ce courant s'inspire notamment des travaux critiques de la britannique Maureen Cain (1979). Celle-ci montre que la majeure partie de l'activité des lawyers est consacrée à traduire en termes juridiques la demande de leurs clients. Le processus de traduction opéré par les professionnels du droit serait ainsi un processus de création parce que ceux-ci inventent le langage dans lequel ils traduisent les demandes émanant de leurs clients.

22. "For some, thinking of law and politics in the same sentence is difficult, especially those who are more familiar with the Continental European approach to law and courts [...]. However, political scientists who study the courts and law in the United States will readily admit that our system had always merged and intertwined these two concepts » (Miller, 2009, p. 1).

23. "We urged to guard against turning [our] bounded observations of law into universals. This approach urges attention to the specific situations, institutions, and struggles out of which the field of Law \& Society emerged» (Silbey et Sarat, 1987, p. 170).

24. D'autres travaux portent sur les conditions d'importation des travaux nord-américains sur les professions juridiques dans les pays d'Europe continentale (Israël et Pélisse, 2004). L'analyse des conditions d'exportation de ces travaux au-delà des frontières nationales est limitée ici aux pays dits occidentaux et, plus particulièrement, d'Europe continentale. Elle ne s'attache donc pas à déterminer les critères à prendre en considération dans le cadre d'études portant sur des pays en voie de développement ou des régimes non démocratiques. À ce sujet, voir : (Ellmann, 1998 ; Krishnan, 2003 ; de Sousa Santos et Rodríguez Garavito, 2005).

25. «In common law countries, boundaries between law and politics tend to be readily permeable. [...] Moreover, the relative autonomy of courts allows cause lawyers, like lawyers in general, to fashion litigation into an assertive political weapon. [...] In civil law systems [...] courts are less independent and more reluctant to protect and extend rights or to impinge on state policies and prerogatives. As a result the boundary between law and politics is more clearly etched than in the common law » (Sarat et Scheingold, 1998, p. 6). 
26. Roscoe Pound, fondateur d'un courant de pensée appelé Sociological jurisprudence est le premier à utiliser cette expression pour se référer aux professionnels du droit nord-américains (Pound, 1967).

27. John Nettl affirme également que le peu d'attention porté à l'État par les sciences sociales américaines est lié à la faiblesse de l'État aux États-Unis : "the relative "statelessness" of American social science coincides with the relative statelessness of the United States» (Nettl, 1968, p. 561).

28. Certains parlent même d'une convergence des modèles juridiques (Felstiner, 2005).

\section{RÉSUMÉS}

Depuis longtemps, les sciences sociales nord-américaines s'interrogent sur la dimension politique de l'activité des professionnels du droit alors qu'en Europe continentale, les rapports entre droit et politique n'ont été analysés conjointement que très récemment et de façon marginale. L'un des enjeux actuels des travaux nord-américains est de transposer les conclusions éprouvées aux États-Unis dans d'autres contextes juridiques et politiques, ce qui questionne l'universalisation et la globalisation de ces analyses. Après avoir exposé les raisons qui expliquent l'intérêt ancien des sciences sociales face à la question du politique aux États-Unis, cet article propose de réfléchir aux conditions et enjeux de l'importation de ces travaux en Europe continentale, en portant une attention particulière aux rapports historiques, et spécifiques à chaque contexte national, entre droit, pouvoir politique et sciences sociales.

For many years, North-American social sciences have been analyzing legal professionals as political actors, while in Continental Europe the relationship among law, politics, and society has remained under-examined. At the moment, a central project for US sociolegal studies is exporting to other political and legal contexts hypotheses previously tested inside US borders, raising the question of the generalizability and/or the globalization of US socio-legal analyses. After briefly describing why social sciences have been focusing on law and social changes in the United States, this article aims to determine what prerequisites are necessary for exporting sociolegal studies outside the US, devoting particular attention to historically contingent -and nationally-distinct- relations between law, political power and the social sciences.

\section{INDEX}

Keywords : Cause Lawyering, Political Activism, Europe, Judicial Politics, Law and Society, Lawyer, Political Lawyering, Realism, Sociology of Law, United States

Mots-clés : Engagement, Professionnels du droit, Réalisme, Sociologie du droit, États-Unis, Europe

\section{AUTEUR}

\section{AUDE LEJEUNE}

Université de Liège, ISHS, 7, boulevard du Rectorat, bâtiment B31, 4000 Liège, Belgique

Aude.Lejeune[at]ulg.ac.be 